\title{
Juvenile methylphenidate reduces prefrontal cortex plasticity via D3 receptor and BDNF in adulthood
}

\author{
Susan L. Andersen ${ }^{1,2 *}$ and Kai C. Sonntag ${ }^{2}$ \\ ' Laboratory for Developmental Neuropharmacology, McLean Hospital and Harvard Medical School, Belmont, MA, USA \\ 2 Department of Psychiatry, McLean Hospital and Harvard Medical School, Boston, MA, USA
}

\author{
Edited by: \\ Nancy Ip, The Hong Kong University \\ of Science and Technology, \\ Hong Kong \\ Reviewed by: \\ Monica Diluca, University of \\ Milano, Italy \\ Zhengping Jia, The Hospital for Sick \\ Children and University of Toronto, \\ Canada \\ Xiaoting Wang, Duke University, \\ USA

\section{*Correspondence:} \\ Susan L. Andersen, Laboratory of \\ Developmental Neuropharmacology, \\ Mailstop 333, 115 Mill Street, \\ McLean Hospital, Belmont, \\ MA 02478, USA \\ e-mail: sandersen@ \\ mclean.harvard.edu
}

Background: Early drug intervention in childhood disorders aims to maximize individual potential in the short- and long-term. Consistently, juvenile exposure to psychostimulants, such as methylphenidate (MPH), reduces risk for substance use in animals and sub-populations of individuals with attention deficit hyperactivity disorder (ADHD). We investigated the effects of MPH on brain plasticity via dopamine receptor D3 (D3R) and brain-derived neurotrophic factor (BDNF) expression in developing rats.

Methods: Between postnatal days 20-35, rat pups were administered saline vehicle (Veh) or MPH $(2 \mathrm{mg} / \mathrm{kg})$, the D3R-preferring agonist \pm 7 -OHDPAT, or the antagonist nafadotride $(0.05 \mathrm{mg} / \mathrm{kg})$ alone, or in combination with MPH twice a day. In adulthood, subjects were challenged to Veh or cocaine $(10 \mathrm{mg} / \mathrm{kg}$ for two days). The prefrontal cortex was analyzed for protein and mRNA levels of total BDNF, its splice variants I, IIc, III/IV, and IVNI, and D3 receptors. A separate group of subjects was assessed for splice variants at $20,35,40$, and 60 days of age.

Results: Across age strong correlations were evident between Drd3 and Bdnf mRNA levels $(r=0.65)$ and a negative relationship between Drd3 and exon Ilc after MPH treatment $(r=-0.73)$. BDNF protein levels did not differ between Veh- and MPH subjects at baseline, but were significantly lower in $\mathrm{MPH}$-treated and cocaine challenged subjects $(30.3 \pm 9.7 \%)$. Bdnf mRNA was significantly higher in MPH-treated subjects, and reversed upon exposure to cocaine. This effect was blocked by nafadotride. Furthermore, Bdnf total and Bdnf splice variants I, IIc, III/IV, and IV/VI changed across the transitions between juvenility and late adolescence.

Conclusions: These data suggest a sensitive window of vulnerability to modulation of BDNF expression around adolescence, and that compared to normal animals, juvenile exposure to MPH permanently reduces prefrontal BDNF transcription and translation upon cocaine exposure in adulthood by a D3R-mediated mechanism.

Keywords: ADHD, adolescence, child, methylphenidate, ritalin, sensitive period

\section{INTRODUCTION}

Children with Attention Deficit Hyperactivity Disorder (ADHD) often respond favorably to psychostimulant therapy, contributing to its use by $61 \%$ of children with ADHD between the ages of 6-13 years (Dalsgaard et al., 2013). The most commonly prescribed stimulant is methylphenidate (MPH). MPH predominantly affects the dopamine and noradrenergic systems, with recent evidence showing a role of the dopamine D3 receptor (D3R) in ADHD-associated behaviors in animals (Andersen et al., 2008; Barth et al., 2013). For example, D3R antagonists reduce hyperactivity and facilitate object recognition in DAT knockout mice, which have been used to model an ADHD phenotype. Other studies further localize D3R actions in object recognition to the prefrontal cortex (Watson et al., 2012).

Childhood/juvenile exposure to psychostimulants during this time of elevated plasticity produces effects that are opposite of those observed in drug-exposed adult animals (Brandon et al., 2001; Dow-Edwards and Busidan, 2001; Andersen et al., 2002;
Bolanos et al., 2003; Andersen, 2005). For example, juvenile, but not adult, exposure to cocaine reduces drug seeking later in life (Dow-Edwards and Busidan, 2001; Andersen et al., 2002). Similarly, juvenile MPH decreases Drd3 mRNA in the prefrontal cortex (PFC) (Andersen et al., 2008), in contrast to an increase in the same region in adult, stimulant-exposed animals (Le Foll et al., 2005). While most adult studies have focused on D3R changes in the nucleus accumbens (Everitt and Robbins, 2000; Le Foll et al., 2005), the reduction in D3R following juvenile MPH exposure is not evident in that region (Andersen et al., 2008). Rather, the juvenile MPH effect on D3R and an MPH-induced place aversion was recapitulated by juvenile treatment with the D3R agonist \pm 7 -OHDPAT (Andersen et al., 2008). Finally, microinjections of \pm 7 -OHDPAT into the PFC reversed aversion, resulting in a preference for cocaine-associated environments.

Cue responsiveness to drug-associated contexts depends on neuroplasticity associated with brain-derived neurotrophic factor (BDNF) levels in adult animals within the prelimbic (pl) PFC 
(Berglind et al., 2007). Adult BDNF levels are transiently elevated following an acute injection of cocaine in both the PFC and the nucleus accumbens (NAc), with sustained elevations of $B d n f$ and Drd3 mRNA found 60 days later in the NAc (Le Foll et al., 2005). Juvenile exposure to MPH reduces $B d n f$ mRNA in the striatum and the hypothalamus with no change in the cingulate cortex during peri-adolescence (Chase et al., 2007). Similar findings of no change in $B d n f$ mRNA were evident in the ventral tegmental area both immediately after treatment and long-term (Warren et al., 2011). However, the enduring effects of $\mathrm{MPH}$ exposure interact with development to manifest fully later in life (Andersen, 2005; Brenhouse and Andersen, 2011). Therefore, the lack of changes in BDNF expression as observed by Chase et al. (2007) in the PFC and Warren et al. (2011) in the ventral tegmental area could have been undetectable, because the window of observation was too early. In order to investigate the time course of D3 receptors and BDNF expression in the developing PFC, we examined the postnatal expression of these two indices and their inter-relationship (Experiment 1). We also examined how previous exposure to MPH may or may not modify BDNF levels to cocaine later in life (Experiment 2). As we suspect that D3 receptors modify BDNF expression in the medial PFC, we manipulated D3 activity in vivo using juvenile exposure to $\mathrm{MPH}$, \pm 7 -OHDPAT, and the D3R antagonist nafadotride (Experiment 3 ). Ultimately, we were interested in how juvenile exposure to $\mathrm{MPH}$ or a D3R-preferring agonist modulates BDNF expression later in life at baseline and following a 2-day unbiased place conditioning paradigm to $10 \mathrm{mg} / \mathrm{kg}$ cocaine.

Environmental influences, such as drug exposure during sensitive periods, can further modulate synaptic structure by altering the expression of Bdnf splice variants (Boulle et al., 2012). Such specific modulation of splice variants permits both spatial and temporal regulation of BDNF expression that in turn, can lead to the precise building of synaptic structure and respond to environmental demands. Understanding how specific splice variants of the Bdnf gene change across age may provide insight into the nature of maturation of different parts of the neuron. Exon I and II are specific to neurons, whereas exons III and IV are found in non-neural tissue as well (Nakayama et al., 1994). The exon I splice variant is expressed primarily in the soma and dendrites, whereas exon II is predominantly in the dendrites, and exon IV is restricted to the soma (Boulle et al., 2012).

Empirical data suggest that different exons are altered following experiences in a semi-unique manner. For example, exon II seems to be preferentially regulated in reward circuits (McCarthy et al., 2012). Sadri-Vakili et al. (2010) demonstrated that chronic cocaine increased $B d n f$ exon IV in the PFC of adult rats. Exon IV contains the binding sites for CREB and $\mathrm{MeCP} 2$ and plays a role in cognitive processes and learning and memory-such as those required for encoding drug-associated cues (Boulle et al., 2012; McCarthy et al., 2012). While BDNF changes have already been associated with addiction-related alterations in structure and function, the majority of these studies have been performed following adult drug exposure paradigms. We are particularly interested in whether exon IV changes following juvenile exposure to MPH, which we have shown increases CREB expression (Andersen et al., 2002). To investigate whether stimulants have unique effects on $B d n f$ splice variants, we determined how $B d n f_{\text {total, }}$ and different $5^{\prime}$ exons splice variants (exons I, IIc, III/IV, and IV/VI), change across age within the late maturing PFC (Experiment 4). Here, we used quantitative Real-Time PCR (qRT-PCR) to examine changes in $B d n f$ exons, as protein-labeling antibodies for the specific exons are not yet available.

\section{MATERIALS AND METHODS SUBJECTS}

Lactating female Sprague-Dawley rats obtained from Charles River were housed with their litters on a $12 \mathrm{~h} / 12 \mathrm{~h}$ light/dark cycle with lights on at 07:00 h with food and water provided ad libidum. Litters were culled to 8 pups of equal numbers of males and females on postnatal day 1 (P1), and were weaned at P21. Care was taken to distribute each condition to one male from each litter. Only males were used for these studies, with the remaining littermates used in other studies. The overall experimental design is found in Figure 1.

\section{DRUGS}

$\mathrm{MPH} \mathrm{HCl}$ (d,l-MPH), \pm 7-OHDPAT, nafadotride, and cocaine $\mathrm{HCl}$ were obtained from Sigma (St. Louis, MO). Drugs were dissolved in $0.9 \%$ saline (vehicle) and administered in a volume of $1 \mathrm{ml} / \mathrm{kg}$. Our earlier study demonstrated no behavioral difference with i.p. vs. oral administration in place conditioning (Brenhouse et al., 2009).

\section{qRT-PCR}

qRT-PCR was performed as described (Andersen et al., 2008). Briefly, brains were rapidly dissected into the mPFC. Sections were snap-frozen in TriReagent solution (Sigma) and stored at $-80^{\circ} \mathrm{C}$ until further analysis. Samples were homogenized, total RNA extracted, DNAse digested (Ambion, Austin, TX), and reverse transcribed into cDNA using the SuperScript II

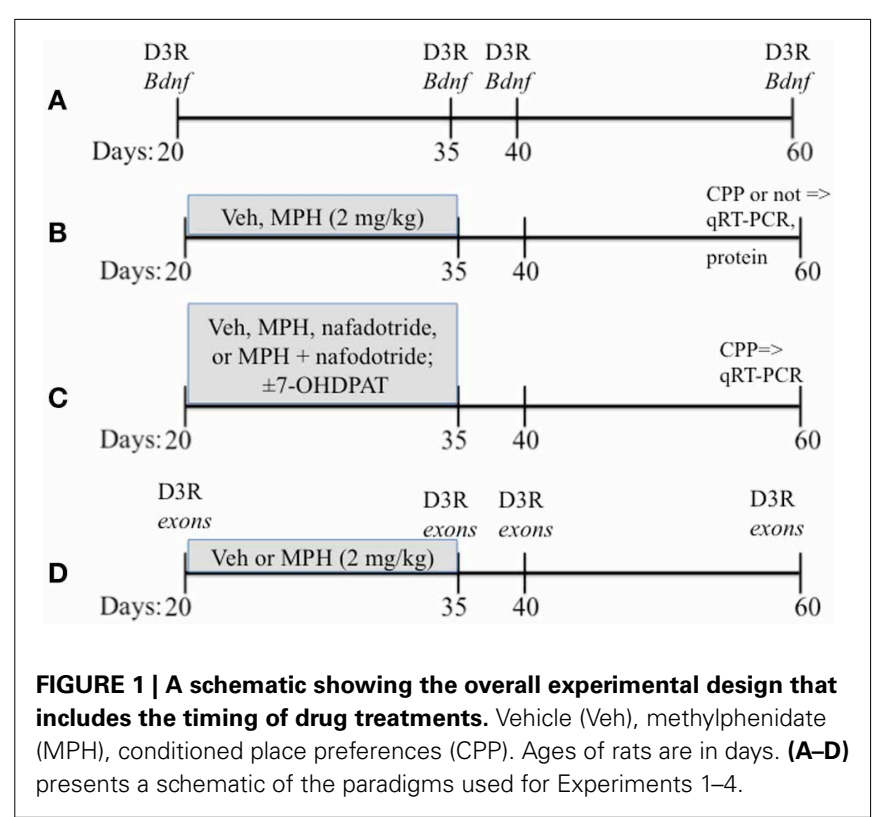


First-Strand Synthesis System (Life Technologies, Foster City, CA). cDNA samples were analyzed by qPCR using the iQ SYBR Green Supermix (BioRad). Amplifications were performed in a total volume of $20 \mu \mathrm{l}$ with $40 \mathrm{nMol}$ primers for each reaction on an Opticon MJ Thermocycler; MJ Research (Watertown, MA). The following published primer sequences were used: $B d n f$ exons

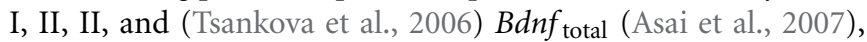
and Drd3 and Gapdh (Andersen et al., 2008).

$B d n f_{\text {total }}:$ F: $5^{\prime}$-ACTCTGGAGAGCGTGAATGG-3' R: $5^{\prime}$-TAC TGTCACACACGCTCAGC-3'

Exon I: F: 5'-CCTGCATCTGTTGGGGAGAC-3' R: 5'-GCC TTGTCCGTGGACGTTTA- $3^{\prime}$

Exon II: F: 5' -CTAGCCACCGGGGTGGTGTAA-3' R: 5'-AGGA TGGTCATCACTCTTCTC- $3^{\prime}$

Exon III F: 5'-CTTCCTTGAGCCCAGTTCC-3' R: $5^{\prime}$-CCGTGG ACGTTTACTTCTTTC-3'

Exon IV F: 5'-CAGAGCAGCTGCCTTGATGTT-3' R: 5'-GCC TTGTCCGTGGACGTTTA-3'

Drd3: F: 5'-AAGCGCTACTACAGCATCTGC-3' R: 5'-GGATAA CCTGCCGTTGCTGAG-3'

Gapdh: F: 5'-AACTCCCATTCTTCCACCTTTG-3' R: 5'-CCC TGTTGCTGTAGCCATATTC-3'

The $B d n f$ exon primers were verified by sequence alignment using deposited sequences in GenBank: Exon I, transcript variant I (Accession EF125675); Exon II: transcript variant IIc (Accession EF125678); Exon III: transcript variant III (Accession EF125686); Exon IV: transcript variant IV (Accession EF125679). A new nomenclature for $B d n f$ transcript variants has recently been suggested by Aid et al. (2007). Accordingly, our primer sequences for exon II, III, and IV amplify new exons IIc, IV, and VI, respectively. Thus, for each exon both the old and the new nomenclature are indicated.

\section{DESIGN}

\section{Experiment 1: The relationship between Drd3 and Bdnf mRNA} IN VIVO

Subjects and Design. mRNA from the PFC was assessed to determine the nature of the relationship between $B d n f_{\text {total }}$ and $D r d 3$ across age. Rats were sacrificed at 4 ages: P25, 35, 40, and 60 representing juvenile, early adolescent, mid adolescent and late adolescent stages respectively (Figure 1A). The PFC was rapidly ( $<1 \mathrm{~min}$ ) dissected out onto chilled glass plates. The sample was then processed for qRT-PCR as described in detail above (3.3). An $n=6$ for P25 and $n=5$ for P35, 40, and 60 were used.

\section{Experiment 2: Measurement of Bdnf total $m R N A$ and BDNF protein levels following MPH treatment with and without cocaine conditioning}

Drug treatment paradigm. Subjects were weighed daily at 09:00 h and injected with either MPH $(2 \mathrm{mg} / \mathrm{kg}$ ) or saline vehicle (Veh) between P20-35 (see Figure 1B; Andersen et al., 2002, 2008. The second injection was administered $4 \mathrm{~h}$ later at 13:00 h. The dose of MPH was selected on the basis of previous studies (Andersen et al., 2002), and approximates a clinically relevant dose in humans based on plasma levels (Wargin et al., 1983;
Kuczenski and Segal, 2002). After P35, the rats received no further drug treatment until behavioral testing or sacrifice for the pharmacological studies 25 days later at P60.

Design. To determine whether PFC BDNF levels and MPH exposure interacted with place conditioning to cocaine, we examined mRNA and protein levels. Drug-exposed subjects were housed with littermates and grown to P60, when they either (1) remained naïve to cocaine exposure (cocaine-naïve) or 2 underwent place conditioning to cocaine $(10 \mathrm{mg} / \mathrm{kg}$, i.p.). The $10 \mathrm{mg} / \mathrm{kg}$ dose of cocaine was chosen based on prior studies (Andersen et al., 2002; Carlezon et al., 2003) that demonstrated a clear differentiation of place conditioning effects between Veh animals. Our earlier study with these animals showed that the Veh subjects demonstrated a minimal preference for cocaine-associated environment, whereas the MPH animals demonstrated a significant place aversion (Andersen et al., 2008). Two sets of subjects were run: one set for $B d n f$ mRNA $(n=6)$ and the second set for BDNF protein $(n=5)$.

Cocaine place conditionin. Unbiased place conditioning, according to standard laboratory methods (Andersen et al., 2002; Carlezon et al., 2003), occurred in a 3-chamber apparatus. This chamber consisted of two large $(24 \times 18 \times 33)$ side compartments separated by a small $(12 \times 18 \times 33 \mathrm{~cm})$ middle compartment. Screening was conducted for $30 \mathrm{~min}$ on day 1 . Rats were placed in the middle compartment and allowed to freely explore the apparatus. Rats that demonstrated a clear preference for either side ( $>18$ of $30 \mathrm{~min}$ ) were eliminated from further testing. Two days of conditioning (with 2 sessions per day) occurred on day 2 and 3 for $60 \mathrm{~min}$, and robust conditioning under these conditions was observed (Andersen et al., 2002; Carlezon et al., 2003). During the conditioning sessions, rats received a $1 \mathrm{ml} / \mathrm{kg}$, i.p. injection of Veh in the morning $(09: 00 \mathrm{~h})$ and confined to one side. The animals were then returned to the home cage. Four hours later, rats received $10 \mathrm{mg} / \mathrm{kg}$ cocaine and confined to the other side. The dose of cocaine for the place conditioning experiments was based on previous results that demonstrated consistent place aversion in MPH-exposed male rats at P60 (Andersen et al., 2002; Carlezon et al., 2003). Sides differed in floor texture, wall colors, and lighting, and assignments were counterbalanced. On day 4, rats were permitted to freely explore the entire apparatus for $30 \mathrm{~min}$ in a drug-free state.

BDNF protein immunoassays. BDNF protein was extracted from the PFC. Dissected tissue was weighed and homogenized in $10 \mathrm{vol} / \mathrm{wt}$ of cold extraction buffer consisting of: $100 \mathrm{mM}$ Tris/ $\mathrm{HCl}, \mathrm{ph}=7.0,2 \%$ bovine serum albumin, $4 \mathrm{mM}$ EDTA, $2 \%$ Triton-X, and the protease inhibitors aprotinin $(5 \mu \mathrm{g} / \mathrm{ml})$, benzamidine $\mathrm{HCl}(157 \mu \mathrm{g} / \mathrm{ml})$, pepstatin A $(0.1 \mu \mathrm{g} / \mathrm{ml})$, and phenylmethylsulfonyl fluoride (PMSF; $17 \mu \mathrm{g} / \mathrm{ml}$ ). Homogenates were centrifuged at $14,000 \mathrm{rpm}$ for $30 \mathrm{~min}$ at $4^{\circ} \mathrm{C}$ and the supernatant was used in the assay. BDNF protein was quantified using the ChemiKine BDNF Sandwich ELISA kit (Chemicon, Temecula, CA) according to the manufacturer's directions. Samples were run in duplicate and quantified 
using standards run simultaneously with the experimental samples.

\section{Experiment 3: Relationship between Bdnf total mRNA and D3R drug treatment}

Subjects. Subjects were exposed to \pm 7 -OHDPAT $(0.3 \mathrm{mg} / \mathrm{kg} ; n=$ 6 ) between P20-35 or Veh ( $n=6$; Figure 1). To further confirm that the effects of MPH were mediated by the D3 receptor, a separate group of subjects was treated with $\mathrm{MPH}(2 \mathrm{mg} / \mathrm{kg}$, i.p.; $n=6)$, nafadotride $(0.05 \mathrm{mg} / \mathrm{kg} ; n=6)$, a combination of MPH and nafadotride (MPH/Nafad; $n=6)$ twice daily between the ages of P20-35 (Andersen et al., 2002).

Design. Subjects were tested with place conditioning to environments associated with $10 \mathrm{mg} / \mathrm{kg}$ cocaine at P60 (Andersen et al., 2008) and sacrificed $24 \mathrm{~h}$ after the last cocaine injection, the PFC rapidly dissected out, and $B d n f$ mRNA changes determined by qRT-PCR (Figure 1C).

\section{Experiment 4: Age and drug effects on the Bdnf splice variants exons I, IIc, III/IV, IV/VI and Drd3}

Subjects. Rats the ages of 20, 35, 40, and 60 days were used in these studies ( $n=6 /$ age). The 20 and 35 days ages were selected to characterize the expression of exons during the drug exposure period, 40 and 60 days represent short- and long-term ages for the paradigm.

Design. The PFC was dissected and processed for mRNA for exons I, IIc, III/IV, IV/VI, and Drd3 across age. Bdnf exons were assessed from subjects exposed to MPH or Veh as juveniles (Figure 1D).

\section{DATA ANALYSIS}

The expression of the $B d n f$ exons was normalized to the housekeeping gene product Gapdh. Linearity ( $r=0.9$ or better) and detection limit of the assay were determined in successive 10fold serial dilutions, performed in triplicate, and an optimal amount of template was chosen for the quantitative analysis. Quantification was performed at a threshold detection line ("threshold cycles," Ct value). The Ct of each gene was normalized against Gapdh, which was run simultaneously for each marker. Expression levels and differences between treatment and Veh groups were determined according to the $2^{-\Delta \mathrm{Ct}}$ or $2^{-\Delta \Delta \mathrm{Ct}}$ method, respectively (Livak and Schmittgen, 2001). Comparisons were made between drug groups with a two-tailed Student's $t$-test or an ANOVA (SPSS); statistical significance was set at $p<0.05$. Data were expressed as mean \pm s.e.m.

\section{RESULTS}

\section{EXPERIMENT 1: THE RELATIONSHIP BETWEEN Drd3 AND Bdnf mRNA IN VIVO}

mRNA from the PFC across age of animals was measured to determine a relationship between $B d n f_{\text {total }}$ and $D r d 3$. Age itself had a significant effect on $\operatorname{Drd} 3\left[F_{(3,17)}=7.87, P<0.001\right]$, but did not significantly influence $B d n f$ mRNA $(p=0.1)$. Regression analysis with ANCOVA showed that Drd3, when co-varied for age, significantly predicted $B d n f$ mRNA $\left[F_{(1,19)}=18.20, P<0.001\right]$ and accounts for $49 \%$ of $B d n f$ changes (Figure 2).
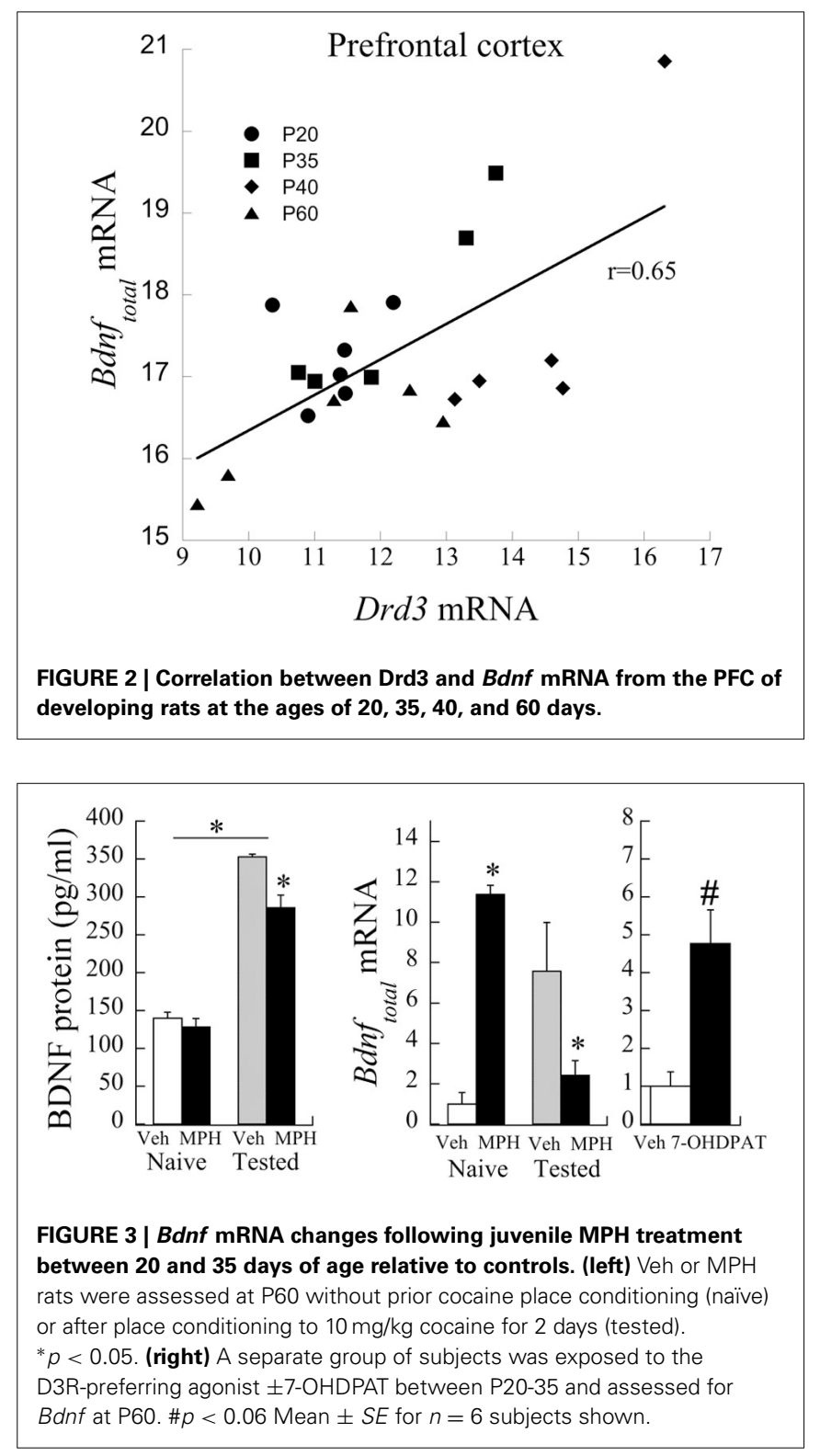

\section{EXPERIMENT 2: Bdnf $_{\text {total }}$ mRNA AND PROTEIN LEVELS FOLLOWING MPH TREATMENT}

The relationship between PFC Bdnf mRNA levels and MPH exposure interacted significantly with whether or not the animals were behaviorally tested with cocaine [Exposure $\times$ Testing interaction: $\left.F_{(1,12)}=6.01, P<0.05\right]$. Bdnf mRNA levels were elevated in MPH subjects relative to Veh controls, and this relationship reversed following behavioral testing to cocaine at P60 [Figure 3 (left)]. Similar to the effects of MPH exposure, juvenile treatment with the D3R-preferring agonist \pm 7 -OHDPAT also increased $B d n f$ mRNA [Figure 3 (right)] in behaviorally-naïve subjects.

As observed in the mRNA levels, a significant interaction was observed in BDNF protein levels as a result of cocaine testing [Exposure $\times$ Testing interaction: $F_{(1,29)}=5.35, p<0.05$ ]. Baseline levels of BDNF protein $(\mathrm{pg} / \mathrm{ml})$ did not significantly 
differ between MPH and Veh subjects $\left[t_{(8)}=0.83, p=0.4\right.$; see Figure 3). After cocaine administration at P60, however, BDNF protein levels in Veh subjects increased $253.1 \pm 28.9 \%$ relative to cocaine naïve controls. In contrast, MPH-exposed subjects had a slightly smaller percent increase $(222.2 \pm 24.4 \%)$ relative to cocaine naïve levels, consistent with lower $B d n f$ mRNA levels in this group.

\section{EXPERIMENT 3: RELATIONSHIP BETWEEN $B d n f_{\text {total }}$ mRNA, D3R, AND MPH TREATMENT}

Consistent with the data in Experiment 2, following juvenile $\mathrm{MPH}$ exposure and place conditioning to cocaine, Bdnf mRNA levels were significantly reduced $30.3 \pm 9.7 \%$ when compared to the Veh exposed subjects $\left[t_{(11)}=2.69, p<0.03\right.$; Figure 4]. In contrast, $B d n f$ mRNA significantly increased $35 \%$ following juvenile exposure to the D3R antagonist, nafadotride, when compared with Veh controls $\left[t_{(12)}=2.65, p<0.03\right]$. No significant difference was observed between the Veh and the MPH/Nafad subjects $\left[t_{(13)}=0.74, p>0.4\right]$.

\section{EXPERIMENT 4: AGE AND DRUG EFFECTS ON Bdnf SPLICE VARIANTS I, IIc, III/IV, IV/VI}

\section{Bdnf mRNA exons change across age}

Individual ANOVAs of exons I, IIc, III/IV, IV/VI were run to determine significant changes across Age; only exon IIc and IV/VI were significantly different $\left[F_{(3,20)}=3.35\right.$ and 4.45 , respectively, $p<0.05$; Figure 5]. Exons I and III/IV did not significantly change across maturation $(p>0.6)$, nor did $B d n f$ total $(p<0.14)$.

\section{Bdnf exon IIc and IV/VI mRNA, Drd3, and MPH exposure}

No significant main effect of MPH exposure was evident at P60 $(p>0.7)$ for exon IIc. However, a strong correlation existed between Drd3 and exon IIc [Pearson's $r=-0.73, p<0.01$;

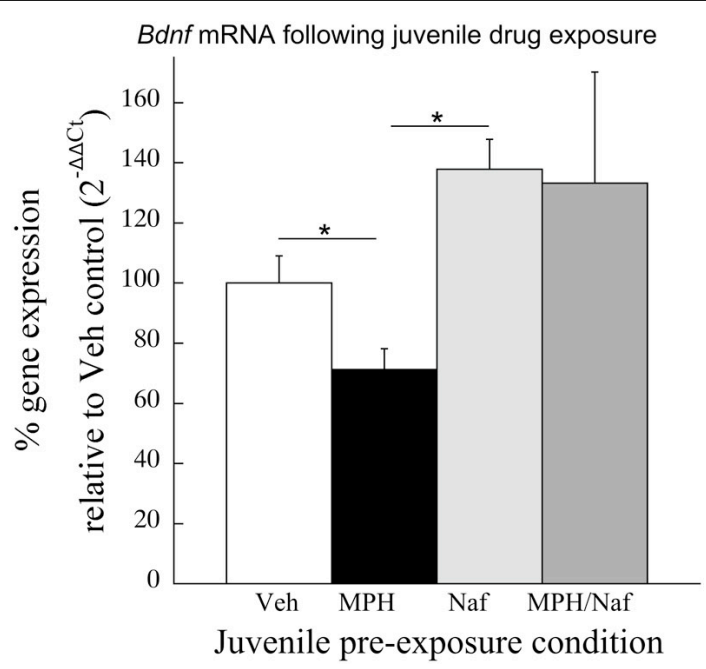

FIGURE 4 | The effects of juvenile treatment with Veh, MPH, the D3R antagonist nafadotride or MPH in combination with nafadotride on Bdnf mRNA levels in the PFC following place conditioning. Means \pm $S E$ are presented for $n=7-8$ subjects/condition. *indicates significant difference at $p<0.05$.
Figure 6 (left)]. Exon IV/VI was significantly reduced following $\mathrm{MPH}$ exposure $\left[F_{(1,10)}=42.43, P<0.001\right]$, as shown in Figure 6 (right), however, this relationship did not correlate with changes in $\mathrm{D} 3$ receptors.

\section{DISCUSSION}

Our data show that juvenile exposure to MPH or manipulation of the D3 dopamine receptor has enduring effects on the expression of BDNF that are related to D3 receptor changes. D3R activation during development did not significantly change BDNF protein levels, but increased $B d n f$ total mRNA while decreasing its splice variants IIc and IV/VI in adulthood. A negative correlation

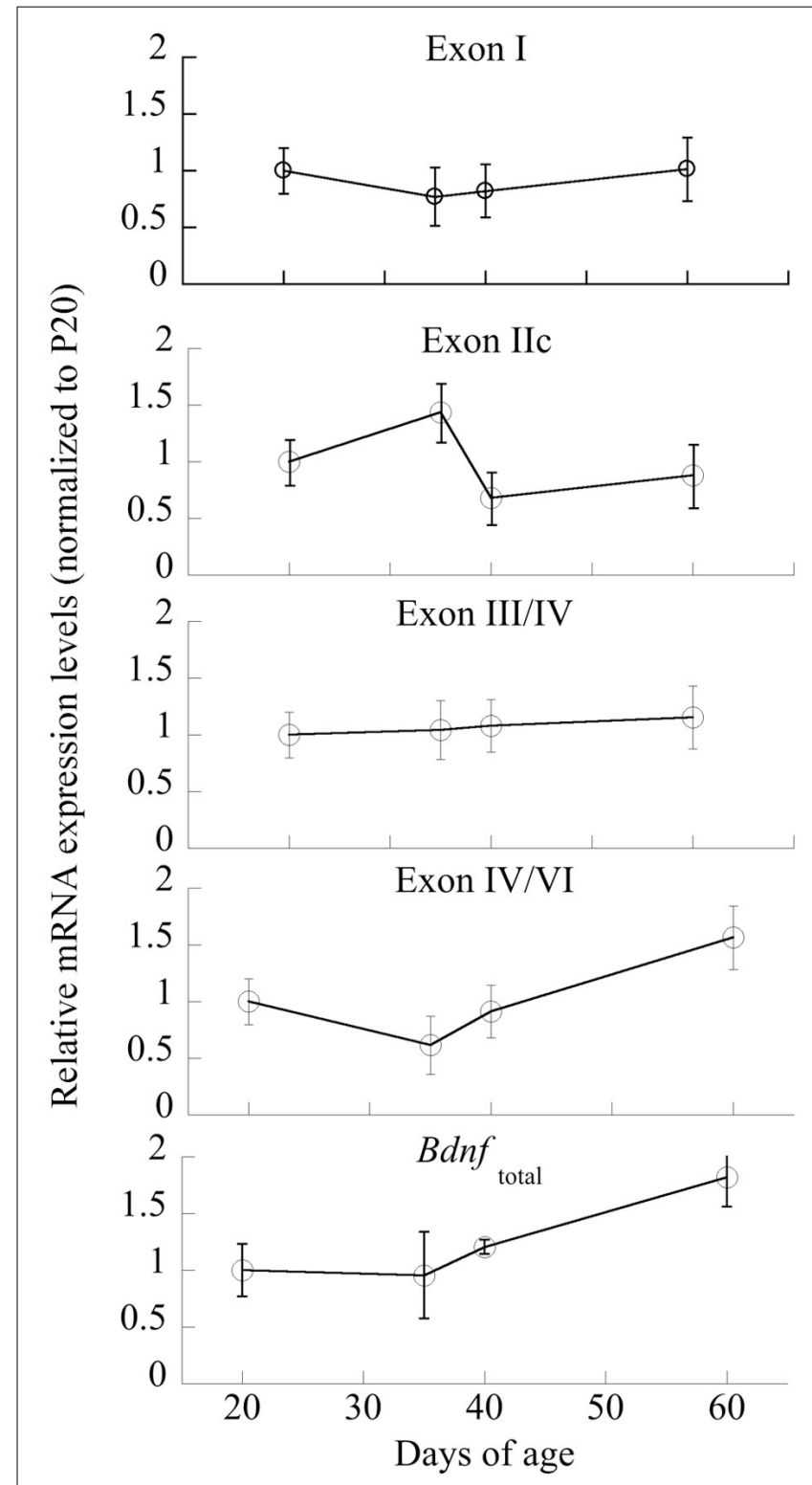

FIGURE 5 | Changes in mRNA expression of Bdnf and its exon-specific splice variants I, Ilc, III/IV, and IV/VI as a function of age. Data are expressed following correction for GAPDH levels run simultaneously. $n=5-6 /$ age, with Means $\pm S E$ presented. 


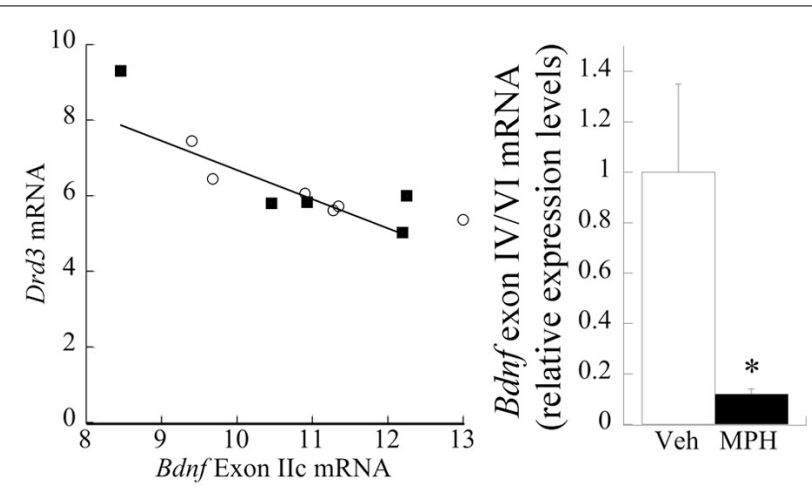

FIGURE 6 | Relationship between D3R, MPH, and BDNF exon splice variants. (left) Drd3 mRNA negatively correlates (Pearson's $r=-0.73$ ) with exon Ilc expression. The filled squares represent the MPH subjects at P60, whereas the open circles are Veh. (right) Juvenile treatment with $\mathrm{MPH}$ significantly reduces exon IVNI expression at 60 days of age relative to the Veh. $n=6 /$ group, with Means $\pm S E$ presented. ${ }^{*} p<0.05$

between $\operatorname{Drd} 3$ and exon IIc mRNA expression was observed. In contrast, a reduction in $B d n f_{\text {total }}$ mRNA and protein were evident in CPP tested animals following D3 receptor activation during development.

$B d n f$ gene regulation occurs in response to environmental stimuli. As D3R antagonism attenuates cue-induced drug-seeking behavior in adult animals (Gal and Gyertyan, 2005), the observed decrease in Drd3 mRNA is consistent with a reduction in place preferences for cocaine-associated cues in animals exposed to $\mathrm{MPH}$ during the juvenile period (Andersen et al., 2002). More importantly, changes in $B d n f$ and its association with Drd3 suggest that the long-term plasticity observed in addiction (Sokoloff et al., 2001) is reduced in MPH animals. Clinically, a subtype of children that is treated with MPH shows reduced substance use disorder (Mannuzza et al., 2008), suggesting that BDNF may play a role.

A sensitive period exists for manipulations of PFC development, with pre-pubertal exposure to psychostimulants offering a window of opportunity for reduced substance abuse disorders (SUD; Andersen, 2005). D3 receptors are localized on dopamine terminals and non-terminal fields (Stanwood et al., 2000), which continue to develop into adolescence (Andersen et al., 2008). During adolescence, regulatory processes in the PFC change markedly (Andersen et al., 1997; Dumont et al., 2004; Tseng and O'Donnell, 2007) that lead to an increase in dopamine function. One of these effects is the loss of a dopamine auto-regulatory-type process mediated by D3 receptors (Booth et al., 1994; Andersen et al., 1997). The current data show that D3R changes across age account for $49 \%$ of the changes in $B d n f$ mRNA, suggesting that D3R may differentially sculpt the pre- vs. post-adolescent PFC by altering neurotrophic expression. Relative to controls, MPHexposed animals show a significant increase in $B d n f_{\text {total }}$ mRNA, but no changes in protein at baseline without challenge. However, $\mathrm{MPH}$ animals had a reduction of $B d n f_{\text {total }} \mathrm{mRNA}$ and protein following cocaine conditioning. BDNF is released with dopamine in the PFC in response to drug cues (Altar et al., 1998; McCarthy et al., 2012). The adult literature shows that cocaine-induced increases of BDNF suppress inhibitory GABAergic interneuron activity in the mPFC [resulting in increased long-term potentiation (LTP) (Lu et al., 2010)]. By extrapolation, a decline in BDNF following juvenile MPH and drug challenge in adulthood would reduce cortical output in response to drug cues relative to controls. A recent study shows that juvenile $\mathrm{MPH}$ reduces the number of spikes in PFC neurons in adulthood-consistent with this hypothesis (Urban et al., 2012).

Modulation of BDNF occurs by histone acetylation or posttranscriptional regulation (Mellios et al., 2008; Caputo et al., 2011; Boulle et al., 2012; Leal et al., 2014), and our data provide evidence for epigenetic modifications in BDNF splice variants by $\mathrm{MPH}$ or D3R. In development, the splice variants II and IV are typically decreased during the transition from juvenile to adolescence. These exons were also reduced in adult animals after juvenile $\mathrm{MPH}$ exposure. A negative correlation between exon IIc and Drd3 mRNA suggests less neuronal plasticity as D3 receptors decrease either following typical aging or that facilitated by juvenile MPH treatment (Andersen et al., 2008). Exon II has been localized to neurons and its expression is activitydependent in visual cortex (Pattabiraman et al., 2005), supporting a potential role in modulating neuronal plasticity. Exon IV/VI was also significantly lower in MPH subjects consistent with previous observations in juvenile MPH rats studied by Fumagalli et al. (2010). It should be noted that the observed up-regulation of $B d n f_{\text {total }}$ mRNA in adult animals after juvenile treatment with MPH did not correlate with BDNF protein levels. There is emerging evidence that protein expression is regulated on the post-transcriptional level by small non-coding RNAs, including micro (mi) RNAs. Several miRNAs have been identified that target the Bdnf transcript (Mellios et al., 2008; Caputo et al., 2011), indicating that other epigenetic mechanisms may be involved in regulating BDNF expression.

The enduring effects in juveniles are predictably opposite to the increase in $B d n f$ exon IV/VI expression in the PFC of adult, cocaine self-administering rats that may underlie craving and relapse (Sadri-Vakili et al., 2010; Schmidt et al., 2013). Exon IV/VI contains the binding sites for CREB and MeCP2 and plays a role in synaptic plasticity, cognitive processes and learning and memory - such as those required for encoding drugassociated cues (McCarthy et al., 2012). While earlier studies have demonstrated reduced cue responsiveness in rats exposed to MPH during the juvenile period (Andersen et al., 2002; Carlezon et al., 2003; Argento et al., 2012), others have shown that periadolescent treatment with MPH reduces object recognition later in life (Leblanc-Duchin and Taukulis, 2007). Interestingly, object recognition increases following D3 receptor blockade in the adult PFC (Watson et al., 2012). Together, these data suggest that MPH may also permanently modulate object recognition later in life. Object recognition measures whether a novel object is recognized, as new or not, which is impaired in ADHD.

Changes in BDNF are important during sensitive period when the programming of drug-seeking behavior occurs (Andersen et al., 2002; Andersen, 2005). Further understanding of the molecular mechanisms regulating these changes can potentially lead to novel ways of harnessing developmental relationships to redirect an off-course trajectory. For example, Shaw et al. (2007) 
have shown that gray matter development of the PFC of ADHD children and teens is delayed. Pharmacological targeting of BDNF itself is difficult, but treatment with a D3R agonist pre-pubertally may reduce behaviors that are associated with ADHD, including risk for SUD, changes in object recognition, and activity levels.

\section{AUTHOR CONTRIBUTIONS}

Both Drs. Andersen and Sonntag contributed equally to the design, execution, and preparation of the manuscript.

\section{ACKNOWLEDGMENTS}

This work was supported by DA-016696 and DA-015403 and the Simches family (to Susan L. Andersen).

\section{REFERENCES}

Aid, T., Kazantseva, A., Piirsoo, M., Palm, K., and Timmusk, T. (2007). Mouse and rat BDNF gene structure and expression revisited. J. Neurosci. Res. 85, 525-535. doi: 10.1002/jnr.21139

Altar, C. A., Fritsche, M., and Lindsay, R. M. (1998). Cell body infusions of brain-derived neurotrophic factor increase forebrain dopamine release and serotonin metabolism determined with in vivo microdialysis. Adv. Pharmacol. 42, 915-921. doi: 10.1016/S1054-3589(08)60896-0

Andersen, S. L. (2005). Stimulants and the developing brain. Trends Pharmacol. Sci. 26, 237-243. doi: 10.1016/j.tips.2005.03.009

Andersen, S. L., Arvanitogiannis, A., Pliakas, A. M., Leblanc, C., and Carlezon, W. A. Jr. (2002). Altered responsiveness to cocaine in rats exposed to methylphenidate during development. Nat. Neurosci. 5, 13-14. doi: $10.1038 / \mathrm{nn} 777$

Andersen, S. L., Dumont, N. L., and Teicher, M. H. (1997). Developmental differences in dopamine synthesis inhibition by 7-OHDPAT. Naunyn Schmiedebergs Arch. Pharmacol. 356, 173-181. doi: 10.1007/PL00005038

Andersen, S. L., Napierata, L., Brenhouse, H. C., and Sonntag, K. C. (2008). Juvenile methylphenidate modulates reward-related behaviors and cerebral blood flow by decreasing cortical D3 receptors. Eur. J. Neurosci. 27, 2962-2972. doi: 10.1111/j.1460-9568.2008.06254.x

Argento, J. K., Arvanitogiannis, A., and Flores, C. (2012). Juvenile exposure to methylphenidate reduces cocaine reward and alters netrin-1 receptor expression in adulthood. Behav. Brain Res. 229, 202-207. doi: 10.1016/j.bbr.2012.01.008

Asai, N., Abe, T., Saito, T., Sato, H., Ishiguro, S., and Nishida, K. (2007). Temporal and spatial differences in expression of TrkB isoforms in rat retina during constant light exposure. Exp. Eye Res. 85, 346-355. doi: 10.1016/j.exer.2007.05.010

Barth, V., Need, A. B., Tzavara, E. T., Giros, B., Overshiner, C., Gleason, S. D., et al. (2013). In vivo occupancy of dopamine D3 receptors by antagonists produces neurochemical and behavioral effects of potential relevance to attention-deficit-hyperactivity disorder. J. Pharmacol. Exp. Ther. 344, 501-510. doi: 10.1124/jpet.112.198895

Berglind, W. J., See, R. E., Fuchs, R. A., Ghee, S. M., Whitfield, T. W. Jr., Miller, S. W., et al. (2007). A BDNF infusion into the medial prefrontal cortex suppresses cocaine seeking in rats. Eur. J. Neurosci. 26, 757-766. doi: 10.1111/j.14609568.2007.05692.x

Bolanos, C. A., Barrot, M., Berton, O., Wallace-Black, D., and Nestler, E. J. (2003). Methylphenidate treatment during pre- and periadolescence alters behavioral responses to emotional stimuli at adulthood. Biol. Psychiatry 54, 1317-1329. doi: 10.1016/S0006-3223(03)00570-5

Booth, R. G., Baldessarini, R. J., Marsh, E., and Owens, C. E. (1994). Actions of (+/-)-7-hydroxy-N,N-dipropylaminotetralin (7-OH-DPAT) on dopamine synthesis in limbic and extrapyramidal regions of rat brain. Brain Res. 662, 283-288. doi: 10.1016/0006-8993(94)90827-3

Boulle, F., Van Den Hove, D. L., Jakob, S. B., Rutten, B. P., Hamon, M., Van Os, J., et al. (2012). Epigenetic regulation of the BDNF gene: implications for psychiatric disorders. Mol. Psychiatry 17, 584-596. doi: 10.1038/mp.2011.107

Brandon, C. L., Marinelli, M., Baker, L. K., and White, F. J. (2001). Enhanced reactivity and vulnerability to cocaine following methylphenidate treatment in adolescent rats. Neuropsychopharmacology 25, 651-661. doi: 10.1016/S0893$133 \mathrm{X}(01) 00281-0$
Brenhouse, H. C., and Andersen, S. L. (2011). Developmental trajectories during adolescence in males and females: a cross-species understanding of underlying brain changes. Neurosci. Biobehav. Rev. 35, 1687-1703. doi: 10.1016/j.neubiorev.2011.04.013

Brenhouse, H. C., Napierata, L., Kussmaul, L., Leussis, M., and Andersen, S. L. (2009). Juvenile methylphenidate exposure and factors that influence incentive processing. Dev. Neurosci. 31, 95-106. doi: 10.1159/000207498

Caputo, V., Sinibaldi, L., Fiorentino, A., Parisi, C., Catalanotto, C., Pasini, A., et al. (2011). Brain derived neurotrophic factor (BDNF) expression is regulated by microRNAs miR-26a and miR-26b allele-specific binding. PLoS ONE 6:e28656. doi: 10.1371/journal.pone.0028656

Carlezon, W. A. Jr., Mague, S. D., and Andersen, S. L. (2003). Enduring behavioral effects of early exposure to methylphenidate in rats. Biol. Psychiatry 54, 1330-1337. doi: 10.1016/j.biopsych.2003.08.020

Chase, T., Carrey, N., Soo, E., and Wilkinson, M. (2007). Methylphenidate regulates activity regulated cytoskeletal associated but not brain-derived neurotrophic factor gene expression in the developing rat striatum. Neuroscience 144, 969-984. doi: 10.1016/j.neuroscience.2006.10.035

Dalsgaard, S., Nielsen, H. S., and Simonsen, M. (2013). Five-fold increase in national prevalence rates of attention-deficit/hyperactivity disorder medications for children and adolescents with autism spectrum disorder, attentiondeficit/hyperactivity disorder, and other psychiatric disorders: a danish register-based study. J. Child Adolesc. Psychopharmacol. 23, 432-439. doi: 10.1089/cap.2012.0111

Dow-Edwards, D. L., and Busidan, Y. (2001). Behavioral responses to dopamine agonists in adult rats exposed to cocaine during the preweaning period. Pharmacol. Biochem. Behav. 70, 23-30. doi: 10.1016/S0091-3057(01)00582-2

Dumont, N. L., Andersen, S. L., Thompson, A. P., and Teicher, M. H. (2004). Transient dopamine synthesis modulation in prefrontal cortex: in vitro studies. Brain Res. Dev. Brain Res. 150, 163-166. doi: 10.1016/j.devbrainres.2004.02.010

Everitt, B. J., and Robbins, T. W. (2000). Second-order schedules of drug reinforcement in rats and monkeys: measurement of reinforcing efficacy and drug-seeking behaviour. Psychopharmacology 153, 17-30. doi: 10.1007/ s002130000566

Fumagalli, F., Cattaneo, A., Caffino, L., Ibba, M., Racagni, G., Carboni, E., et al. (2010). Sub-chronic exposure to atomoxetine up-regulates BDNF expression and signalling in the brain of adolescent spontaneously hypertensive rats: comparison with methylphenidate. Pharmacol. Res. 62, 523-529. doi: 10.1016/j.phrs.2010.07.009

Gal, K., and Gyertyan, I. (2005). Dopamine D3 as well as D2 receptor ligands attenuate the cue-induced cocaine-seeking in a relapse model in rats. Drug Alcohol Depend. 81, 63-70. doi: 10.1016/j.drugalcdep.2005.05.011

Kuczenski, R., and Segal, D. S. (2002). Exposure of adolescent rats to oral methylphenidate: preferential effects on extracellular norepinephrine and absence of sensitization and cross-sensitization to methamphetamine. J. Neurosci. 22, 7264-7271.

Leal, G., Comprido, D., and Duarte, C. B. (2014). BDNF-induced local protein synthesis and synaptic plasticity. Neuropharmacology 76, 639-656. doi: 10.1016/j. neuropharm.2013.04.005

Leblanc-Duchin, D., and Taukulis, H. K. (2007). Chronic oral methylphenidate administration to periadolescent rats yields prolonged impairment of memory for objects. Neurobiol. Learn. Mem. 88, 312-320. doi: 10.1016/j.nlm.2007. 04.010

Le Foll, B., Diaz, J., and Sokoloff, P. (2005). A single cocaine exposure increases BDNF and D3 receptor expression: implications for drug-conditioning. Neuroreport 16, 175-178. doi: 10.1097/00001756-200502080-00022

Livak, K. J., and Schmittgen, T. D. (2001). Analysis of relative gene expression data using real-time quantitative PCR and the 2(-Delta Delta $\mathrm{C}(\mathrm{T})$ ) method. Methods 25, 402-408. doi: 10.1006/meth.2001.1262

Lu, H., Cheng, P. L., Lim, B. K., Khoshnevisrad, N., and Poo, M. M. (2010). Elevated BDNF after cocaine withdrawal facilitates LTP in medial prefrontal cortex by suppressing GABA inhibition. Neuron 67, 821-833. doi: 10.1016/j.neuron.2010.08.012

Mannuzza, S., Klein, R. G., Truong, N. L., Moulton, J. L. 3rd., Roizen, E. R., Howell, K. H., et al. (2008). Age of methylphenidate treatment initiation in children with ADHD and later substance abuse: prospective follow-up into adulthood. Am. J. Psychiatry 165, 604-609. doi: 10.1176/appi.ajp.2008.07091465

McCarthy, D. M., Brown, A. N., and Bhide, P. G. (2012). Regulation of BDNF expression by cocaine. Yale J. Biol. Med. 85, 437-446. 
Mellios, N., Huang, H. S., Grigorenko, A., Rogaev, E., and Akbarian, S. (2008). A set of differentially expressed miRNAs, including miR-30a-5p, act as posttranscriptional inhibitors of BDNF in prefrontal cortex. Hum. Mol. Genet. 17, 3030-3042. doi: 10.1093/hmg/ddn201

Nakayama, M., Gahara, Y., Kitamura, T., and Ohara, O. (1994). Distinctive four promoters collectively direct expression of brain-derived neurotrophic factor gene. Brain Res. Mol. Brain Res. 21, 206-218. doi: 10.1016/0169-328X(94) 90251-8

Pattabiraman, P. P., Tropea, D., Chiaruttini, C., Tongiorgi, E., Cattaneo, A., and Domenici, L. (2005). Neuronal activity regulates the developmental expression and subcellular localization of cortical BDNF mRNA isoforms in vivo. Mol. Cell. Neurosci. 28, 556-570. doi: 10.1016/j.mcn.2004.11.010

Sadri-Vakili, G., Kumaresan, V., Schmidt, H. D., Famous, K. R., Chawla, P., Vassoler, F. M., et al. (2010). Cocaine-induced chromatin remodeling increases brainderived neurotrophic factor transcription in the rat medial prefrontal cortex, which alters the reinforcing efficacy of cocaine. J. Neurosci. 30, 11735-11744. doi: 10.1523/JNEUROSCI.2328-10.2010

Schmidt, H. D., McGinty, J. F., West, A. E., and Sadri-Vakili, G. (2013). Epigenetics and psychostimulant addiction. Cold Spring Harb. Perspect. Med. 3: a012047. doi: 10.1101/cshperspect.a012047

Shaw, P., Eckstrand, K., Sharp, W., Blumenthal, J., Lerch, J. P., Greenstein, D., et al. (2007). Attention-deficit/hyperactivity disorder is characterized by a delay in cortical maturation. Proc. Natl. Acad. Sci. U.S.A. 104, 19649-19654. doi 10.1073/pnas.0707741104

Sokoloff, P., Le Foll, B., Perachon, S., Bordet, R., Ridray, S., and Schwartz, J. C. (2001). The dopamine D3 receptor and drug addiction. Neurotox. Res. 3 433-441. doi: 10.1007/BF03033202

Stanwood, G. D., Artymyshyn, R. P., Kung, M. P., Kung, H. F., Lucki, I., and McGonigle, P. (2000). Quantitative autoradiographic mapping of rat brain dopamine D3 binding with [(125)I]7-OH-PIPAT: evidence for the presence of D3 receptors on dopaminergic and nondopaminergic cell bodies and terminals. J. Pharmacol. Exp. Ther. 295, 1223-1231.

Tsankova, N. M., Berton, O., Renthal, W., Kumar, A., Neve, R. L., and Nestler, E. J. (2006). Sustained hippocampal chromatin regulation in a mouse mode of depression and antidepressant action. Nat. Neurosci. 9, 519-525. doi: $10.1038 / \mathrm{nn} 1659$
Tseng, K. Y., and O’Donnell, P. (2007). Dopamine modulation of prefrontal cortical interneurons changes during adolescence. Cereb. Cortex 17, 1235-1240. doi: 10.1093/cercor/bhl034

Urban, K. R., Waterhouse, B. D., and Gao, W. J. (2012). Distinct age-dependent effects of methylphenidate on developing and adult prefrontal neurons. Biol. Psychiatry 72, 880-888. doi: 10.1016/j.biopsych.2012.04.018

Wargin, W., Patrick, K., Kilts, C., Gualtieri, C. T., Ellington, K., Mueller, R. A. et al. (1983). Pharmacokinetics of methylphenidate in man, rat and monkey. J. Pharmacol. Exp. Ther. 226, 382-386.

Warren, B. L., Iniguez, S. D., Alcantara, L. F., Wright, K. N., Parise, E. M., Weakley, S. K., et al. (2011). Juvenile administration of concomitant methylphenidate and fluoxetine alters behavioral reactivity to reward- and mood-related stimuli and disrupts ventral tegmental area gene expression in adulthood. J. Neurosci. 31, 10347-10358. doi: 10.1523/JNEUROSCI.1470-11.2011

Watson, D. J., Loiseau, F., Ingallinesi, M., Millan, M. J., Marsden, C. A., and Fone, K. C. (2012). Selective blockade of dopamine D3 receptors enhances while D2 receptor antagonism impairs social novelty discrimination and novel object recognition in rats: a key role for the prefrontal cortex. Neuropsychopharmacology 37, 770-786. doi: 10.1038/npp.2011.254

Conflict of Interest Statement: The authors declare that the research was conducted in the absence of any commercial or financial relationships that could be construed as a potential conflict of interest.

Received: 03 October 2013; accepted: 03 January 2014; published online: 21 January 2014.

Citation: Andersen SL and Sonntag KC (2014) Juvenile methylphenidate reduces prefrontal cortex plasticity via D3 receptor and BDNF in adulthood. Front. Synaptic Neurosci. 6:1. doi: 10.3389/fnsyn.2014.00001

This article was submitted to the journal Frontiers in Synaptic Neuroscience.

Copyright (c) 2014 Andersen and Sonntag. This is an open-access article distributed under the terms of the Creative Commons Attribution License (CC BY). The use, distribution or reproduction in other forums is permitted, provided the original author(s) or licensor are credited and that the original publication in this journal is cited, in accordance with accepted academic practice. No use, distribution or reproduction is permitted which does not comply with these terms. 